\title{
Condições de vida e mortalidade infantil no município do Embu, São Paulo
}

\author{
Living conditions and infant mortality in the municipality of Embu, São Paulo, Brazil
}

Renato Nabas Ventura ${ }^{1}$, Eleonora Menicucci de Oliveira², Edina Mariko K. da Silva ${ }^{3}$, Nilza Nunes da Silva ${ }^{4}$, Rosana Fiorini Puccini ${ }^{5}$

\section{RESUMO}

Objetivo: Descrever a distribuição do coeficiente de mortalidade infantil e seus componentes no município do Embu, São Paulo, no período de 1995 a 1998, segundo os estratos de condições de vida.

Métodos: Estudo descritivo com análise por conglomerados, dos 135 setores censitários do município de Embu, agrupados em quatro estratos de condições de vida: estrato 1, com melhores condições de moradia, renda e escolaridade; estratos 2 e 3, intermediários; estrato 4, no qual todas as moradias eram aglomerados subnormais ou favelas. Foram calculados os coeficientes de mortalidade infantil, neonatal e pós-neonatal, a proporção entre óbitos neonatais e pós-neonatais, o risco atribuível populacional e mortalidade proporcional por causas, para os anos de 1995 a 1998, segundo os quatro estratos de condições de vida estabelecidos.

Resultados: $\mathrm{O}$ estrato 4 apresentou maiores coeficientes de mortalidade infantil e risco atribuível populacional em relação aos estratos intermediários, em todos os anos do estudo. Esse estrato apresentou, também, as menores proporções entre mortalidade neonatal e pós-neonatal. O risco atribuível populacional no estrato 4 foi maior que os demais estratos para as afecções perinatais $(159,4)$, doenças respiratórias $(271,4)$ e doenças infecciosas $(415,6)$. Identificaram-se dados demográficos semelhantes em áreas próximas aos limites geograficamente constituídos pelo estudo e heterogeneidade de eventos num mesmo território.
Conclusões: Identificou-se uma relação entre desigualdades sociais e mortalidade infantil, segundo os critérios de condições de vida estabelecidos para este estudo, entretanto, não houve distribuição homogênea nos quatro estratos populacionais, revelando dificuldades em utilizá-los como parâmetros para desigualdades sociais em grandes centros urbanos.

Palavras-chave: mortalidade infantil; desigualdades em saúde; estudos ecológicos; indicadores de saúde; indicadores sociais.

\section{ABSTRACT}

Objective: To describe the infant mortality coefficient and its components in the municipality of Embu, São Paulo, Brazil, between 1995 and 1998, according to strata of living conditions.

Methods: A cross-sectional descriptive study was conducted using cluster analysis in the 135 census session of the municipality of Embu, grouped into four strata of living conditions: stratum 1, with the best living, income and schooling conditions; strata 2 and 3 with regular conditions; and stratum 4 , in which all houses were subnormal agglomerates or slums. The neonatal, post-neonatal and infant mortality rates, the population's ascribable risk, the ratio between neonatal and post-neonatal deaths and the mortality due to ascribable causes were calculated for 1995 to 1998 , for each stratum.
1Doutor em Ciências, Pediatra da disciplina de Pediatria Geral e Comunitária do Departamento de Pediatria da Escola Paulista de Medicina da Universidade Federal de São Paulo (Unifesp-EPM). São Paulo, SP, Brasil

2Professora titular da disciplina de Ciências Humanas em Saúde do Departamento de Medicina Preventiva da Unifesp-EPM. São Paulo, SP, Brasil ${ }^{3}$ Doutora em Ciências, professora adjunta da disciplina de Emergências e Medicina Baseada em Evidência do Departamento de Medicina da UnifespEPM, São Paulo, SP, Brasil

${ }^{4}$ Professora associada do Departamento de Epidemiologia da Faculdade de Saúde Pública da Universidade de São Paulo. São Paulo, SP, Brasil ${ }^{5}$ Professora titular da disciplina de Pediatria Geral e Comunitária, chefe do Departamento de Pediatria da Unifesp-EPM. São Paulo, SP, Brasil
Endereço para correspondência:

Rosana Fiorini Puccini

Rua Botucatu, 598 - Vila Clementino

CEP 04023-062 - São Paulo/SP

E-mail: rpuccini@terra.com.br

Recebido em: 19/3/2208

Aprovado em: 29/6/2008

Fonte financiadora: Fundação de Amparo à Pesquisa do Estado de São Paulo (Fapesp), processo 95/3438-7 
Results: In all studied years, the stratum 4 presented the highest infant mortality rates and higher population's ascribable risk compared to intermediate strata. This stratum has also presented the lowest ratio between neonatal and post-neonatal mortality. The population's attributable risk in the stratum 4 was higher for perinatal affections (159.4), respiratory (271.4) and infectious (415.6) diseases. Similar demographic data in areas close to the geographically limits of the study and heterogeneity of events in the same territory were present.

Conclusions: Relationship between social inequality and infant mortality was identified according to the living conditions criteria. However, heterogeneous distribution was shown within each stratum, precluding its use for evaluating social inequalities in big urban centers.

Key-words: infant mortality; health inequalities; ecological studies; health indicators; social indicators.

\section{Introdução}

Por expressar em conjunto as condições de vida, trabalho e acesso aos serviços de saúde, a mortalidade infantil constitui um dos indicadores utilizados para abordar as desigualdades sociais e a complexa determinação do processo saúde-doença. A análise das condições de saúde, referenciada territorialmente, é um campo de estudo que tem se desenvolvido, colocando em evidência essas desigualdades e possibilitando melhor direcionamento de ações sociais e de saúde $e^{(1-5)}$.

A tendência de redução da mortalidade infantil tem sido verificada no mundo inteiro, entretanto, permanecem profundas desigualdades. Segundo a Organização Mundial de Saúde $(\mathrm{OMS})^{(6)}$ em 2005, a probabilidade de uma criança morrer antes dos cinco anos de idade em Angola e no Afeganistão era de 260 e 257/1.000 nascidos vivos, respectivamente. No Japão, nesse mesmo ano, essa probabilidade era de 4/1.000 e, no Brasil, de 33/1.000 nascidos vivos. As realidades de cada um desses países são diversas, assim como as possibilidades de intervenção. As relações desses indicadores com o desenvolvimento socioeconômico de cada país estão bem estabelecidas, entretanto, outros determinantes estão envolvidos.

No Brasil, a tendência da mortalidade infantil durante o século passado foi de declínio em todas as regiões, acumulando, no período de 1930 a 1990, uma redução de $71 \%$ : de $158,3 / 1.000$ nascidos vivos (1930) para 66,9/1.000 nascidos vivos (1990). Essa redução foi acompanhada de mudanças expressivas nos indicadores demográficos, socioeconômicos e de saúde que, no entanto, ocorreram de forma heterogênea, com diferenças entre os estados municípios ${ }^{(1,7)}$. No período de 1990 a 2004, o coeficiente de mortalidade infantil no Brasil, que era de 48,4/1.000, diminuiu para 22,6/1.000 nascidos vivos, persistindo ainda acentuadas diferenças entre as regiões ${ }^{(8)}$.

Em Embu, município da região metropolitana de São Paulo, o coeficiente de mortalidade infantil até meados da década de 1980 era um dos mais elevados do Estado de São Paulo, mas apresentou redução importante nos anos de 1990 e, em 2006, atingiu o valor de 11,2/1.000 nascidos vivos ${ }^{(9)}$. Essa redução ocorreu nos seus componentes neonatal e pósneonatal, sendo que os óbitos neonatais, na última década, correspondem a cerca de $70 \%$ do total. O melhor acesso às informações e a estruturação do pré-natal de risco na rede básica de Embu, a partir de 1996, explicam, em parte, essa redução da mortalidade. Outros fatores, possivelmente, também contribuíram para diminuir os componentes neonatal e pós-neonatal da mortalidade infantil no município como: redução das taxas de fecundidade, expansão da atenção básica à saúde e de serviços de apoio diagnóstico e terapêutico, desenvolvimento de um programa regular de atenção integral à saúde da criança que proporciona, entre outras ações, a promoção da saúde e a assistência oportuna frente às principais afecções, com destaque às infecções respiratórias. Em 1999, foi criado um hospital geral na região, o que representou grande avanço para a assistência do município, disponibilizando leitos de maternidade, unidade neonatal (média e alta complexidade) e de pediatria. Em 2004, a criação da maternidade municipal complementou essa assistência, garantindo a realização de cerca de $80 \%$ dos partos do Sistema Único de Saúde (SUS) no município.

Frente à persistência de inadequadas condições de vida de um grande contingente da população de Embu, coloca-se a possibilidade de que segmentos populacionais se encontrem em situação de maior vulnerabilidade para morbidade e mortalidade, em especial, a população infantil. Assim, este estudo foi realizado com o objetivo de descrever o coeficiente de mortalidade infantil e seus componentes no município, no período de 1995 a 1998, segundo estratos de condições de vida, visando a oferecer subsídios para os gestores no direcionamento de suas ações e políticas de saúde.

\section{Métodos}

Embu dista $25 \mathrm{~km}$ da capital do Estado, caracterizandose por possuir uma pequena concentração industrial, baixo 
potencial de crescimento econômico e por contar com amplas áreas de proteção de mananciais. O município é uma estância turística, $100 \%$ urbanizado e sua população, em 1996, era de 194.879 habitantes $^{(9)}$. Nos anos de 1995 a 1998, período de realização deste estudo, a Rede Municipal de Saúde era constituída por nove Unidades Básicas de Saúde (UBS), dois Prontos-Socorros (PS) e não havia leitos hospitalares. Desde 1970, a Universidade Federal de São Paulo (Unifesp) desenvolve atividades de ensino, assistência e pesquisa no município, atuando de forma articulada aos gestores locais.

Este é um estudo descritivo que utilizou dados secundários, contidos nas declarações de óbitos de todas as crianças que morreram antes de completar um ano, residentes no município de Embu, nos anos de 1995 a 1998. Os quatro estratos de condições de vida utilizados neste estudo haviam sido definidos, anteriormente, no projeto "Morbidade Infantil e Utilização de Serviços de Saúde”, realizado em 1996 e financiado pela Fundação de Amparo à Pesquisa do Estado de São Paulo (Fapesp). A metodologia da estratificação está detalhada no relatório do projeto original de Silva et $a l^{(10)} \mathrm{e}$ em trabalho de Frei ${ }^{(11)}$.

Os trabalhos desses autores tinham como base metodológica o censo demográfico de 1991, utilizado para cálculo da amostra do projeto original, o qual apontava 18.602 crianças menores de cinco anos no município. A abordagem tomou como fundamentos as situações de risco coletivo, avaliando-se os diferenciais intra-urbanos por meio da construção de indicadores compostos para estudo das condições de vida. Dispondo de arquivos de dados do censo demográfico de 1991, a população foi estratificada segundo as condições de moradia e situação socioeconômica das famílias. Os 135 setores censitários do município foram reunidos por meio da técnica de análise de agrupamentos denominada average linkage. $\mathrm{O}$ tipo de moradia, o número de moradores por domicílio, saneamento básico, renda e escolaridade dos chefes de família foram as variáveis consideradas para discriminar os quatro estratos resultantes: estrato 1, constituído por 17 setores censitários (concentrados na área turística do município) com melhores condições de moradia, saneamento básico, renda e escolaridade; estratos 2 e 3, constituídos por 67 e 34 setores censitários, respectivamente, refletiam as condições ambientais intermediárias e predominantes no município; estrato 4 , no qual todas as moradias foram classificadas como aglomerados subnormais ou favelas, com piores condições de saneamento básico, renda e escolaridade.
Tabela 1 - Número de nascidos vivos e óbitos em menores de um ano no município de Embu, São Paulo, nos anos 1995 a 1998

\begin{tabular}{ccc}
\hline Ano & Nascidos Vivos & $\begin{array}{c}\text { Óbitos de menores } \\
\text { de um ano }\end{array}$ \\
\hline 1995 & 3.711 & 130 \\
1996 & 3.948 & 111 \\
1997 & 3.922 & 106 \\
1998 & 4.846 & 104 \\
\hline
\end{tabular}

Fonte: Fundação Seade

Os dados foram obtidos das declarações de óbitos de menores de um ano residentes em Embu e fornecidas pela Fundação Sistema Estadual de Análise de Dados (Seade). Os dados de 1995 foram obtidos do estudo de Araújo ${ }^{(12)}$. Nos anos de 1995 a 1998 ocorreram 130,111, 106 e 104 óbitos de crianças menores de um ano, respectivamente (Tabela 1). A seguir, procedeu-se à geolocalização dos mesmos, segundo os quatro estratos de condições de vida, calculando-se os coeficientes de mortalidade infantil, neonatal e pós-neonatal para cada estrato/ano. Para o cálculo destes coeficientes, o número de nascidos vivos considerado para cada estrato foi obtido utilizando-se a distribuição percentual da população geral presente em cada um dos quatro estratos. Foi utilizado, também, o risco atribuível populacional (RAP) proposto por Nunes $e t a l^{(13)}$, que é a diferença relativa proporcional entre cada uma das unidades de análise (neste estudo, o coeficiente de mortalidade infantil/ano/estrato) e um valor de referência (coeficiente de mortalidade infantil de Embu/ano). Esta medida visa avaliar o impacto que teria, sobre o indicador considerado, a redução da desigualdade existente entre cada valor da unidade de análise e o valor de referência que pode ser tomado como meta para orientar intervenções. Para a análise da distribuição dos óbitos por causas determinadas, segundo os estratos, foram somados os óbitos ocorridos nos anos de 1996 a 1998 (total de 321 óbitos). Não foram localizadas cinco declarações de óbito, sendo duas de 1996, uma de 1997 e duas de 1998. Este estudo foi aprovado pelo Comitê de Ética em Pesquisa da Unifesp.

\section{Resultados}

$\mathrm{Na}$ Tabela 2, observa-se que o estrato 4 apresentou os maiores coeficientes de mortalidade infantil e neonatal (exceto no ano de 1995) e que, nos estratos 2 e 3, os mesmos coeficientes foram menores que no estrato 1 , contrariando o esperado. Os coeficientes de mortalidade pós-neonatal 
Tabela 2 - Coeficientes de mortalidade infantil, neonatal e pós-neonatal e proporção entre mortalidade neonatal e pós-neonatal e risco atribuível populacional, segundo estratos de condições de vida, no município de Embu, São Paulo, nos anos de 1995 a 1998.

\begin{tabular}{|c|c|c|c|c|c|c|c|c|c|c|c|c|}
\hline \multirow[t]{2}{*}{ Estratos } & \multicolumn{4}{|c|}{ Mortalidade infantil } & \multicolumn{4}{|c|}{ Mortalidade neonatal } & \multicolumn{4}{|c|}{$\begin{array}{l}\text { Mortalidade } \\
\text { pós-neonatal }\end{array}$} \\
\hline & 95 & 96 & 97 & 98 & 95 & 96 & 97 & 98 & 95 & 96 & 97 & 98 \\
\hline 1 & 40,3 & 55,2 & 55,6 & 35,0 & 36,3 & 30,7 & 55,6 & 25,0 & 4,0 & 24,5 & 0,0 & 10,0 \\
\hline 2 & 32,1 & 21,3 & 21,4 & 17,4 & 19,8 & 11,6 & 14,2 & 12,5 & 12,3 & 9,7 & 7,2 & 4,9 \\
\hline 3 & 14,4 & 29,8 & 25,4 & 17,8 & 5,6 & 19,5 & 13,9 & 9,3 & 9,8 & 10,3 & 11,6 & 8,4 \\
\hline 4 & 47,4 & 76,3 & 72,3 & 65,5 & 17,2 & 38,1 & 29,8 & 44,8 & 30,1 & 38,1 & 42,6 & 20,7 \\
\hline Total & 35,0 & 28,1 & 27,0 & 21,5 & 20,5 & 15,7 & 17,3 & 14,4 & 14,5 & 12,4 & 9,7 & 7,0 \\
\hline \multirow[t]{2}{*}{ Estratos } & \multicolumn{6}{|c|}{$\begin{array}{l}\text { Proporção entre mortalidade neonatal } \\
\text { e pós-neonatal }\end{array}$} & \multicolumn{6}{|c|}{ Risco atribuível populacional (RAP) } \\
\hline & \multicolumn{2}{|l|}{95} & 96 & \multicolumn{2}{|c|}{$97^{*}$} & 98 & \multicolumn{2}{|c|}{95} & 96 & \multicolumn{2}{|l|}{97} & 98 \\
\hline 1 & \multicolumn{2}{|c|}{9,0} & 1,3 & \multicolumn{2}{|l|}{-} & 2,5 & \multicolumn{2}{|c|}{15,1} & 96,3 & \multicolumn{2}{|l|}{105,7} & 63,1 \\
\hline 2 & \multicolumn{2}{|c|}{1,6} & 1,2 & \multicolumn{2}{|c|}{2,0} & 2,6 & \multicolumn{2}{|c|}{$-8,3$} & $-24,3$ & \multicolumn{2}{|l|}{20,7} & 19,2 \\
\hline 3 & \multicolumn{2}{|c|}{0,5} & 1,9 & \multicolumn{2}{|c|}{1,2} & 1,1 & \multicolumn{2}{|c|}{$-58,8$} & 6,1 & \multicolumn{2}{|l|}{6,0} & 17,2 \\
\hline 4 & \multicolumn{2}{|c|}{0,5} & 1,0 & \multicolumn{2}{|c|}{0,7} & 2,2 & \multicolumn{2}{|c|}{35,3} & 171,2 & \multicolumn{2}{|l|}{167,6} & 205,3 \\
\hline Total & \multicolumn{2}{|c|}{1,4} & 1,3 & \multicolumn{2}{|c|}{1,8} & 2,0 & \multicolumn{2}{|l|}{-} & - & \multicolumn{2}{|l|}{-} & - \\
\hline
\end{tabular}

* em 1997 não ocorreram óbitos pós-neonatais no estrato 1

Tabela 3 - Causa de morte em menores de um ano, segundo os estratos de condições de vida no município de Embu, São Paulo, no ao de 1996 a 1998.

\begin{tabular}{|c|c|c|c|c|c|c|c|c|c|c|}
\hline \multirow[t]{2}{*}{ Estratos } & \multicolumn{2}{|c|}{$\begin{array}{l}\text { Afecções } \\
\text { perinatais }\end{array}$} & \multicolumn{2}{|c|}{$\begin{array}{c}\text { Doenças } \\
\text { respiratórias }\end{array}$} & \multicolumn{2}{|c|}{$\begin{array}{l}\text { Doenças } \\
\text { infecciosas }\end{array}$} & \multicolumn{2}{|c|}{ Outras causas } & \multicolumn{2}{|c|}{ Total de óbitos } \\
\hline & $\mathrm{n}$ & $\% *$ & $n$ & $\% *$ & $n$ & $\%^{*}$ & $n$ & $\%^{*}$ & $\mathrm{n}$ & $\% *$ \\
\hline 1 & 16 & $61,5(9,2)$ & 0 & $0,0(0,0)$ & 2 & $7,7(15,3)$ & 8 & $38,8(8,6)$ & 26 & $100,0(8,2)$ \\
\hline 2 & 99 & $57,6(56,9)$ & 23 & $13,4(63,9)$ & 3 & $1,7(23,1)$ & 47 & $27,3(50,5)$ & 172 & $100,0(54,4)$ \\
\hline 3 & 32 & $50,8(18,4)$ & 5 & $7,9(13,9)$ & 4 & $6,3(30,8)$ & 22 & $34,3(23,7)$ & 63 & $100,0(19,9)$ \\
\hline 4 & 27 & $49,1(15,5)$ & 8 & $14,5(22,2)$ & 4 & $7,3(30,8)$ & 16 & $29,1(17,2)$ & 55 & $100,0(17,5)$ \\
\hline Total & 174 & $55,1(100,0)$ & 36 & $11,4(100,0)$ & 13 & $4,1(100,0)$ & 93 & $29,4(100,0)$ & 316 & $100,0(100,0)$ \\
\hline
\end{tabular}

${ }^{*}$ O percentual antes dos parênteses corresponde ao número/total de cada estrato e o percentual entre parênteses corresponde ao número/total de cada causa de morte

apresentaram uma tendência inversa às condições de vida da população de cada estrato, com exceção do estrato 1 (1996 e 1998) e dos estratos 2 e 3 (1995). A proporção entre óbitos neonatais e pós-neonatais apresentou uma tendência decrescente em relação aos estratos (do estrato 1 para o 4) nos anos de 1995 e 1997. O RAP evidenciou a contribuição do estrato 4 para a mortalidade infantil do município, seguido do estrato 1 , em todos os anos do estudo.

A porcentagem de óbitos por afecções perinatais, no período de 1996 a 1998, foi maior no estrato $1(61,5 \%)$ e diminuiu à medida que pioravam as condições de vida, chegando a 49,1\% no estrato 4 (Tabela 3). Na mesma tabela, verificou-se que as doenças infecciosas mostraram tendência inversa. Ao analisar-se o RAP de mortalidade por causas, observou-se que, no estrato 4 , encontraram-se os maiores índices $(159,4)$, comparados à média do município. Índices também desfavoráveis ocorreram no estrato 1 , comparado aos estratos 2 e 3 (Tabela 4). O RAP para mortalidade por doenças respiratórias e para doenças infecciosas foi muito superior no estrato 4 (Tabela 4). 
Tabela 4 - Coeficiente de mortalidade infantil (CMI) por mil nascidos vivos (NV) por afecções perinatais, doenças respiratórias e doenças infecciosas, segundo os estratos de condições de vida no município de Embu, São Paulo, no ano de 1996 a 1998.

\begin{tabular}{lcccccc}
\hline Estratos & \multicolumn{2}{c}{ Afeç̧ões perinatais } & \multicolumn{2}{c}{ Doenças respiratórias } & \multicolumn{2}{c}{ Doença infecciosas } \\
\cline { 2 - 7 } & CMI/1000NV & $\begin{array}{c}\text { Risco } \\
\text { atribuível } \\
\text { populacional }\end{array}$ & CMI/1000NV & $\begin{array}{c}\text { Risco } \\
\text { atribuível } \\
\text { populacional }\end{array}$ & CMI/1000NV & $\begin{array}{c}\text { Risco } \\
\text { atribuível } \\
\text { populacional }\end{array}$ \\
\hline 1 & 30,5 & 122,8 & 0,0 & 0,0 & 3,8 & 272,5 \\
2 & 11,5 & $-16,1$ & 2,7 & $-5,7$ & 0,4 & $-65,7$ \\
3 & 11,4 & $-17,7$ & 1,8 & $-37,1$ & 1,4 & 39,2 \\
4 & 35,5 & 159,4 & 10,5 & 271,4 & 5,3 & 415,6 \\
\hline Total & $\mathbf{1 3 , 7}$ & - & $\mathbf{2 , 8}$ & - & $\mathbf{1 , 0}$ & - \\
\hline
\end{tabular}

\section{Discussão}

Algumas considerações devem ser feitas para interpretar os resultados obtidos: primeiro, foi constatada grande heterogeneidade interna nos estratos, mais acentuada no estrato 1 devido à presença de caseiros (com baixos salários, escolaridade e condições de moradia) e de moradores de condomínios de alto padrão; segundo, foi instalado um empreendimento imobiliário de padrão superior à média do município no estrato 3 entre o ano de 1991 (quando foi realizado o censo cujos dados foram utilizados para a estratificação) e os anos de 1995 a 1998 (período considerado no estudo); terceiro, o estrato 4, constituído por favelas, não se concentra prioritariamente em uma única área do município, verificando-se a existência de núcleos de moradias subnormais em outros estratos; quarto, o óbito infantil é um evento pouco freqüente, sobretudo no estrato 1 com menor proporção da população, fazendo com que uma única ocorrência possa determinar grandes variações de coeficiente; quinto, o número de nascidos vivos por estrato foi calculado em função da distribuição da população geral, quando é conhecido o fato de que a população de maior renda apresenta menores taxas de fecundidade. Num primeiro momento, estes fatos podem ter determinado resultados não esperados.

A partir do conceito da determinação social do processo saúde-doença ${ }^{(14,15)}$, esperava-se que os setores com piores condições de vida apresentassem os maiores coeficientes de mortalidade infantil, a menor proporção entre mortalidade neonatal e pós-neonatal, maiores valores de risco atribuível populacional, menor contribuição em relação à mortalidade por causas perinatais e maior em relação às causas infecciosas e parasitárias. Os resultados deste estudo confirmaram em parte essas hipóteses. A estratificação, a partir de indicadores compostos, buscou delinear no município as áreas homogê- neas quanto às condições de vida da população e este objetivo não foi alcançado plenamente. $\mathrm{O}$ emprego dos indicadores compostos por diferentes variáveis socioeconômicas permitiu uma aproximação com as condições materiais de residentes dos grupos populacionais existentes no município de Embu. Entretanto, verificou-se não haver distribuição homogênea nas áreas com melhores condições de vida, intermediárias e com piores condições de vida, expondo as dificuldades para utilizar este tipo de estratificação para desigualdades sociais em áreas urbanas. Foram também identificados dados demográficos semelhantes em áreas próximas aos limites geograficamente constituídos pelo estudo. Um dos pontos discutidos junto aos gestores do município de Embu foi que a estratificação estabelecida, com base nos setores censitários, não apresenta correspondência com as áreas de abrangência das unidades básicas de saúde. Muitas ações são programadas com base nos indicadores de saúde da área correspondente a cada UBS, considerando aspectos geográficos e de acessibilidade. A territorialização para o gestor é um processo dinâmico, pois deve responder com agilidade às mudanças na ocupação e, a cada nova UBS, a população adstrita deve ser também redistribuída.

Bezerra-Fillho et a ${ }^{(16)}$ afirmam que regiões vizinhas tendem a apresentar mais similaridades que regiões distantes e que, à medida que o acesso ao saneamento básico e à educação melhoram, os valores médios ficam muito semelhantes e perdem o poder de discriminação espacial. Estudos que analisam a dependência espacial da mortalidade infantil e de seus componentes têm apresentado resultados diversos e até conflitantes. Inúmeros fatores devem ser considerados na interpretação desses resultados como o desenvolvimento do país ou região, o porte do município, os patamares do coeficiente e de seus componentes, as causas e a metodologia empregada para estratificação. 
A expressão da complexidade dessa análise pôde ser evidenciada com os resultados encontrados neste estudo para o estrato 1 , que reflete a convivência dos dois pilares da desigualdade social num mesmo espaço geográfico. De um lado, os proprietários de chácaras, casas em condomínios de alto padrão; de outro, os caseiros que convivem nesses mesmos espaços, porém, com dificuldades de acesso aos serviços de saúde e relação precária de trabalho, agregando ao salário, o direito à moradia e ao cultivo de subsistência. Embora desfavorável, essa condição se apresenta, ainda, com algumas vantagens em relação ao estrato 4 , dispondo, em geral, de moradias de melhor qualidade, com menor número de pessoas por cômodo, e produção (mesmo que parcial) do próprio alimento.

Verificou-se que o coeficiente de mortalidade pós-neonatal apresentou relação com as condições de vida, evidenciada por valores crescentes a partir do estrato 2 , exceto no ano de 1995. Andrade et al ${ }^{(17)}$, em estudo realizado no Paraná, concluem que o componente pós-neonatal pode discriminar os diferentes segmentos populacionais, não se encontrando essa mesma relação para o componente neonatal. Tal relação, entretanto, foi observada por outros autores que utilizaram o índice de desenvolvimento humano (IDH) no agrupamento de municípios ${ }^{(18)}$.

A análise da mortalidade neonatal tem se mostrado bastante complexa. Ela agrega um grupo de óbitos evitáveis e, também, óbitos inevitáveis ou menos influenciados pelas condições de vida, quando comparados ao componente pós-neonatal. As afecções perinatais, principal grupo de causas desse componente, relacionam-se mais diretamente à assistência pré-natal, à atenção ao parto e à assistência ao recém-nascido, que, hoje, está com acesso ampliado em grandes centros urbanos ${ }^{(16)}$, às malformações congênitas e anomalias cromossômicas que, segundo grupo de causas, são caracteristicamente de difícil intervenção em todos os segmentos sociais. No presente estudo, a proporção de óbitos do grupo afecções perinatais se reduziu de acordo com a estratificação, chegando a uma participação de 49,1\% nos óbitos de menores de um ano no estrato 4. O RAP, por esta causa, entretanto, que considera o coeficiente por mil nascidos vivos, foi superior nesse mesmo estrato.

Tem sido observado, neste e em outros trabalhos, uma aproximação do coeficiente de mortalidade neonatal entre as classes sociais, porém, seus determinantes são diferentes. Nas classes sociais de maior poder aquisitivo, o aumento decorre das gestações múltiplas devido às técnicas de reprodução assistida, maior idade materna com prevalências elevadas de hipertensão arterial e diabetes, resultando em gestação e recém-nascidos de maior risco para morbimortalidade e, ainda, às altas taxas de ce- sáreas, comprovadamente associadas a agravos maternos e para o recém-nascido ${ }^{(19,20)}$. O aumento ou estagnação, nas últimas duas décadas, do número de recém-nascidos com baixo peso e prétermo em diferentes regiões, incluindo as mais desenvolvidas, corroboram essas hipóteses ${ }^{(21-23)}$. Na população de baixa renda, por outro lado, os estudos apontam para outros determinantes da mortalidade neonatal: restrição de crescimento intra-uterino devido à desnutrição materna, falta de acesso aos serviços de pré-natal e assistência ao parto de qualidade, resultando em prematuridade evitável, peregrinação da gestante no momento do parto, dentre outros ${ }^{(24-26)}$.

Os resultados deste estudo, concluído em 1999, foram apresentados aos gestores e ao Conselho Municipal de Saúde, constituindo subsídio importante para o redirecionamento das ações. Esse processo foi favorecido com a criação do hospital geral, que disponibilizou serviço de pré-natal e leitos para gestantes de alto risco, recursos diagnósticos e serviço de neonatologia para médio e alto risco, complementado, em 2004, com a Maternidade de Embu, permitindo a organização do sistema de referência e contra-referência. Merece destaque o agendamento realizado pela maternidade da primeira consulta para o binômio mãe-recémnascido na UBS nos primeiros quinze dias de vida da criança e, ainda, o encaminhamento para ambulatório multiprofissional, no próprio município, de recém-nascidos pré-termo ou com peso ao nascer inferior a $1.500 \mathrm{~g}$, também por ocasião da alta da maternidade. Em 2004, foi criado o Comitê de Mortalidade Infantil com o objetivo de investigar todos os óbitos infantis evitáveis e identificar os pontos de estrangulamento na assistência como pré-natal, parto, assistência neonatal e ao lactente. A classificação dos óbitos como evitáveis tem como base o conceito de evento-sentinela, introduzido por Rutstein et al ${ }^{(27)}$ e refere-se a uma ocorrência que poderia ser prevenida, dada a disponibilidade de tecnologia médica suficiente para evitá-la ou algo que não deveria ocorrer se o serviço de saúde funcionasse adequadamente. Nos patamares atuais, com aproximadamente 50 óbitos por ano no município, o conceito de óbitos evitáveis ${ }^{(28)}$ e a discussão com a equipe de profissionais têm proporcionado uma atuação mais efetiva. Embora este estudo tenha sido realizado em um único município da região metropolitana de São Paulo, seus indicadores sociais e sua realidade de saúde em muito se assemelham às condições de periferias de grandes centros urbanos. A discussão da territorialização e desta experiência de intervenção podem representar uma contribuição para a compreensão da mortalidade infantil, limites e possibilidades das ações de saúde.

Mesmo reconhecendo a importância das ações de caráter público direcionadas ao controle e redução da mortalidade infantil, 
é necessário reduzir as desigualdades sociais como forma de sustentabilidade aos ganhos conquistados e para estabelecer condições para novos avanços ${ }^{(29,30)}$. A mortalidade infantil permanece como mais uma manifestação das contradições entre as classes sociais, identificadas na desigualdade de inserção no mundo do trabalho, de condições de vida, acesso à educação e à saúde. A resposta aos índices desfavoráveis desse coeficiente não pode ser, unicamente, o desenvolvimento de uma política de focalização, característica das reformas sociais na América Latina ${ }^{(31)}$, as quais

\section{Referências bibliográficas}

1. Oliveira LAP, Mendes MMS. Mortalidade infantil no Brasil: uma avaliação de tendências recentes. In: Minayo MCS, organizadora. Os muitos Brasis: saúde e população na década de $80.2^{a}$ ed. São Paulo: Hucitec Abrasco; 1999. p. 291-303.

2. Castellanos PL. Epidemiologia, saúde pública, situação de saúde e condições de vida: considerações conceituais. In: Barata RB, organizadora. Condições de vida e situação de saúde. Rio de Janeiro: Abrasco; 1997. p. 31-75.

3. Malta DC, Almeida MCM, Dias MAS, Merhy EE. A mortalidade infantil em Belo Horizonte, Minas Gerais, Brasil, por área de abrangência dos Centros de Saúde (1994-1996). Cad Saude Publica 2001;17:1189-98.

4. Boing $A F$, Boing $A C$. Infant mortality from preventable causes in Brazil: an ecological study in 2000-2002. Cad Saude Publica 2008;24:447-55.

5. Costa MCN, Azi PA, Paim JS, Silva LMV. Mortalidade infantil e condições de vida: a reprodução das desigualdades sociais em saúde na década de 90. Cad Saude Publica 2001;17:555-67.

6. World Health Organization [homepage on the Internet]. Countries [cited 2008 Jun 06]. Available from: http://www.who.int/countries

7. Simões CCS, Monteiro CA. Tendência secular e diferenciais regionais da mortalidade infantil no Brasil. In: Monteiro CA, editor. Velhos e novos males da saúde no Brasil: a evolução do país e suas doenças. $2^{a}$ ed. São Paulo: Hucitec, Nupens/USP; 2000. p. 153-6.

8. Brasil - Ministério da Saúde. [homepage on the Internet] Indicadores e Dados Básicos-Brasil 2006. Indicadores de mortalidade. [cited 2008 Jun 06]. Available from: http://tabnet.datasus.gov.br/cgi/idb2006/matriz.htm\#mort

9. Brasil - Fundação Seade. Fundação Sistema Estadual de Análise de Dados. [homepage on the Internet]. Fundação Sistema Estadual de Análise de Dados. Produtos. Perfil Municipal. Embu [cited 2008 Jun 06]. Available from: http:// www.seade.gov.br/produtos/perfil/perfil.php

10. Silva NN, Puccini RF, Araújo NS, Pedroso GC, Gomes MT, Furlani WJ et al. Morbidade e utilização de serviços de saúde no município de Embu: estudo epidemiológico na área da saúde infantil. São Paulo: Unifesp/USP/Secretaria Municipal de Saúde de Embu; 1997.

11. Frei F. Análise de agrupamentos: estudo metodológico e aplicações em epidemiologia [tese de mestrado]. São Paulo: USP; 1998.

12. Araujo NSA. Contribuições ao gerenciamento local de informações em saúde para o programa de saúde infantil do município do Embu [tese de mestrado]. São Paulo: USP; 1999

13. Nunes A, Santos JR, Barata RB, Viana SM. Medindo as desigualdades em saúde no Brasil: uma proposta de monitoramento. Brasília: Organização Pan-Americana de Saúde, Instituto de Pesquisas Econômicas Aplicadas; 2001.

14. Possas $C$. Epidemiologia e sociedade: heterogeneidade estrutural e saúde no Brasil. São Paulo: Hucitec; 1989.

15. Laurell AC. A saúde-doença como processo social. In: Nunes ED, editor. Medicina social: aspectos históricos e teóricos. São Paulo: Global; 1983. p. 133-58.

16. Bezerra Filho JG, Kerr LR, Miná DL, Barreto ML. Spatial distribution of the infant mortality rate and its principal determinants in the State of Ceará, Brazil, 2000-2002. Cad Saude Publica 2007;23:1173-85. têm adotado medidas meramente compensatórias aos efeitos do ajuste estrutural, em detrimento da universalização dos direitos sociais. Cada óbito ocorrido no primeiro ano de vida deve ser encarado como um problema complexo, um evento final resultante da teia de relações de poder entre classes, tornando explícita a necessidade da compreensão das condições em que ocorreu esse óbito e as formas mais candentes de expressão desta desigualdade presente nos grupos familiares e no território onde vivem e constroem a rede de proteção social ${ }^{(32)}$.

17. Andrade SM, Soares DA, Matsuo T, Souza RK, Mathias TA, Iwakura ML et al Living conditions and infant mortality in the state of Paraná, Brazil, 1997/2001. Cad Saude Publica 2006;22:181-9.

18. Nascimento LF, Batista GT, Dias NW, Catelani CS, Becker D, Rodrigues L. Spatial analysis of neonatal mortality in Paraíba Valley, Southeastern Brazil, 1999 to 2001. Rev Saude Publica 2007;41:94-100.

19. Villar J, Valladares E, Wojdyla D, Zavaleta N, Carroli G, Velazco A; WHO 2005 global survey on maternal and perinatal health research group. Caesarean delivery rates and pregnancy outcomes: the 2005 WHO global survey on maternal and perinatal health in Latin América. Lancet 2006;367:1819-29.

20. MacDorman MF, Declercq E, Menacker F, Malloy MH. Neonatal mortality for primary cesarean and vaginal births to low-risk women: application of an "intention-to-treat" model. Birth 2008;35:3-8.

21. Seque CA, Kawazoe L, Silva EM, Puccini RF. Assistência pré-natal e ao parto de mães de crianças usuárias do serviço de vacinação de unidade de saúde da cidade de são Paulo. Rev Paul Pediatr 2007;25:38-46.

22. Barbieri MA, Silva AA, Bettiol $H$, Gomes UA. Risk factors for the increasing trend in low birth weight among live births born by vaginal delivery, Brazil. Rev Saude Publica 2000;34:596-602.

23. Monteiro CA, Benicio MH, Ortiz LP. Secular trends in birth weight in the city of São Paulo, Brazil (1976-1998). Rev Saude Publica 2000;34(6 Suppl): 26-40.

24. Ferrari LS, Brito AS, Carvalho AB, Gonzáles MR. Neonatal mortality in Londrina, Paraná State, Brazil, in 1994, 1999, and 2002. Cad Saude Pública 2006;22:1063-71.

25. Pereira APE, Gama SGN, Leal MC. Mortalidade infantil em uma amostra de nascimentos do município do Rio de Janeiro, 1999-2001: "linkage" com o Sistema de Informação de Mortalidade. Rev Bras Saude Mater Infant 2007;7:83-8.

26. Schoeps D, Furquim de Almeida M, Alencar GP, França Jr I, Novaes HM Franco de Siqueira AA et al. Risk factors for early neonatal mortality. Rev Saude Publica 2007;41:1013-22.

27. Rutstein DD, Berenberg W, Chalmers TC, Child CG 3rd, Fishman AP, Perrin EB. Measuring the quality of medical care. A clinical method. N Engl J Med 1976;294:582-8.

28. Jobim R, Aerts D. Avoidable infant mortality and associated factors in Porto Alegre, Southern Brazil, 2000-2003. Cad Saude Publica 2008;24: 179-87.

29. Bezerra-Filho JG, Kerr-Pontes LR, Sansgolo LR, Barreto ML. Infant mortality and the sócio-economic scenario in the State of Ceará, Brazil in 1991 and 2001. Rev Bras Saude Matern Infant 2007;7:135-42.

30. Duarte CMR. Health policy effects on infant mortality trends in Brazil: a literature review from the last decade. Cad Saude Publica 2007;23:1511-28.

31. Pereira PA. Necessidades humanas. Subsídios à crítica dos mínimos sociais São Paulo: Cortez; 2000.

32. Silva MO. Introdução. In: Silva MO, editor. Comunidade Solidária: o não enfrentamento da pobreza no Brasil. São Paulo: Cortez; 2001. p. 11-30. 